\title{
Miranda
}

Revue pluridisciplinaire du monde anglophone /

Multidisciplinary peer-reviewed journal on the English-

speaking world

$17 \mid 2018$

Paysages et héritages de David Bowie

\section{Modern Death. La critique rock face à Blackstar}

\section{Claude Chastagner}

\section{(2) OpenEdition}

\section{Journals}

Édition électronique

URL : http://journals.openedition.org/miranda/12525

DOI : $10.4000 /$ miranda. 12525

ISSN : 2108-6559

Éditeur

Université Toulouse - Jean Jaurès

Référence électronique

Claude Chastagner, « Modern Death. La critique rock face à Blackstar », Miranda [En ligne], 17 | 2018, mis en ligne le 20 septembre 2018, consulté le 16 février 2021. URL : http://journals.openedition.org/ miranda/12525; DOI : https://doi.org/10.4000/miranda.12525

Ce document a été généré automatiquement le 16 février 2021.

\section{c) (i)}

Miranda is licensed under a Creative Commons Attribution-NonCommercial-NoDerivatives 4.0 International License. 


\title{
Modern Death. La critique rock face à Blackstar
}

\author{
Claude Chastagner
}

\section{Blackstar, l'œuvre ultime}

1 Il était prévisible que les rock stars vieillissantes, celles du moins qu'un gramme ou un verre de trop n'avaient pas déjà fauchées, allaient bientôt nous quitter. En 2016, ce fut le cas de Glenn Frey, Paul Kantner, Keith Emerson, Prince, Leon Russell, Leonard Cohen, George Michael. Et David Bowie, trois jours après la sortie de son dernier album, Blackstar (auquel la presse fait également référence sous le nom de Black Star).

2 Le précédent, The Next Day, remontait à 2013, sa dernière prestation scénique à 2006, sa dernière interview à 2005. Le 19 novembre 2015, un premier single, «Blackstar ", puis un second, "Lazarus ", le 17 décembre, dix jours après la première de la comédie musicale du même nom co-écrite par Bowie, avaient donné un avant-goût du nouvel album et indiqué un regain de créativité. Blackstar sort le 8 janvier 2016, le jour de ses 69 ans. Le 10 janvier, Bowie décède des suites d'un cancer du foie qui s'était déclaré 18 mois auparavant.

3 Black Star est un succès commercial. Classé numéro un des ventes en Grande-Bretagne dès sa sortie, il reste à cette position trois semaines et n'en est chassé que par un autre album de Bowie, la compilation Best of Bowie (2002). Une semaine après son décès, dixneuf albums de David Bowie sont classés dans la liste des cent meilleures ventes du pays, dont dix dans les quarante premières, pour un total de six cent vingt mille ventes. Black Star est également en tête des ventes plusieurs semaines dans vingt-quatre autres pays, dont les États-Unis, l'Australie, le Canada, la France, l'Allemagne, l'Italie, la Russie, l'Espagne, numéro deux à Taiwan, en Corée du Sud, au Mexique, etc. Les ventes mondiales s'élèvent en juin 2017 à près de deux millions d'unités, tous supports confondus.

4 C'est également un succès critique. The Daily Telegraph lui attribue cinq étoiles sur cinq, quatre pour The Guardian, The Independent, NME, Rolling Stone et AllMusic. Les principales 
publications spécialisées comme généralistes le classent parmi les meilleurs albums de l'année, à la première place pour Rolling Stone, Mojo, Newsweek, Q, Uncut, The Village Voice, au second rang pour The New York Times et Wire. L'agrégateur de critiques Metacritic lui attribue une note globale de 87 sur 100 (ce qui signifie « universal acclaim »), obtenue à partir de la recension de 43 critiques officielles, et une note de $8,4 / 10$ à partir des appréciations de 613 utilisateurs du site. Dans un premier temps, les thématiques plutôt obscures, vaguement inquiétantes de l'album sont perçues comme un commentaire de notre époque, sa violence, la fragmentation et l'obscurantisme religieux qui la caractérisent. Mais si de nombreuses critiques publiées en amont soulignent la tonalité élégiaque, voire mortifère (Pareles), les allusions à la mort ne sont jamais perçues comme pouvant concerner de façon spécifique David Bowie, tous soulignant au contraire son regain d'énergie et de créativité et spéculant avec délectation sur les surprises que pourra recéler l'album suivant.

La mort est un thème récurrent de la chanson populaire, y compris du rock. En revanche, il est plus rare que les auteurs envisagent leur propre départ, lointain ou imminent, que ce soit sur le mode combatif («The Show Must Go On», Queen, 1991), poignant, («'Til I Die », The Beach Boys, 1971, que Brian Wilson imposa contre leur gré aux autres membres du groupe), ou grinçant («I'd Rather Be Dead», Harry Nilsson, 1972). À ces titres nous pourrions ajouter les méditations apaisées, presque amusées du dernier Leonard Cohen, You Want It Darker (2016), les ultimes soubresauts de Johnny Cash refusant de mourir à coups de reprises, au fil de ses American Recordings, III (2000) et IV (2002), le dernier album de Warren Zevon, The Wind. Et Blackstar.

6 Car une fois la mort de David Bowie connue, l'album change de statut. Il devient difficile pour les auditeurs comme pour les critiques professionnels de ne pas le réécouter au prisme de ce décès, de ne pas y déceler les traces d'une mort annoncée, que certains commentaires des singles avant-coureurs avaient pressentie, sans en savoir l'imminence. Comme l'écrit Bester Langs, «Difficile de ne pas regarder le dernier clip de 'Blackstar' (titre génial par ailleurs) en cherchant la maladie derrière le maquillage " (Langs 2016). "Quoi de plus poignant, ajoute Auréliano Tonet dans Le Monde, qu'un artiste qui chante son dernier soupir ?» (Tonet 2016). Les vidéos officielles, la pochette de l'album («aux allures de faire-part», précise l'AFP, 2016), les paroles ou les musiques constituent autant d'éventuels indices ou de messages symboliques dont Bowie aurait parsemé son ultime œuvre. On y décrypte des signes de ce qu'Ann Powers appelle «le courage et l'intuition de Bowie à concevoir sa propre élégie musicale " (Powers 2016). On cite en particulier les paroles de «Lazarus» («Look up here, I’m in heaven / I've got scars that can't be seen »), de « Dollar Days " ("Don't believe for just one second I'm forgetting you / I'm trying to / I'm dying to ») ou le leitmotiv nostalgique et apaisé du dernier morceau, "I Can't Give Everything Away». La chanson-titre, "Blackstar ", évoque pour certains le nom parfois donné aux tumeurs cancéreuses (Vincent 2016, Britton 2016). D'autres y voient une allusion à «Black Star », méditation sur la mort enregistrée en 1960 par Elvis Presley («When a man sees his black star, he knows his time has come »), dont Bowie était un grand admirateur (ils étaient par ailleurs nés le même jour, celui précisément de la sortie de l'album).

7 Je me suis intéressé non pas au contenu de cet ultime album, mais à ces critiques. Mon étude porte sur un ensemble de vingt-cinq chroniques et recensions publiées dans la presse spécialisée et généraliste française ainsi que de quarante-six autres dans la presse britannique et étatsunienne dans les mois qui ont précédé ou suivi le décès de 
David Bowie. Je me suis principalement attaché aux critiques de l'album et des vidéos, plutôt qu'aux notices nécrologiques portant sur la vie et l'œuvre de David Bowie. Qu'ont dit les professionnels comme les amateurs de sa dernière production et dans quelle tonalité ? Élégiaque, hagiographique, critique ? En quoi le décès de l'artiste a-til modifié leur appréciation de cet opus et comment ces éventuelles modifications ontelles été exprimées ? Parlent-ils de l'homme plutôt que du disque ? Entend-on des voix discordantes ou l'expression d'un deuil collectif ? Observe-t-on des divergences de ton et d'appréciation ou au contraire, Bowie fait-il l'unanimité autour de son dernier disque, comme les chiffres de vente semblent le prouver ?

En toute logique temporelle, la recherche universitaire sur le rock, après avoir concentré ses premiers efforts au début des années soixante-dix sur la jeunesse des artistes et du public, s'intéresse aujourd'hui à la fin imminente des représentants de l'âge d'or du genre. À ce titre, je m'interroge sur ce qui est exprimé, via ces critiques, du rapport à la mort au prisme de la musique rock. Car le rock véhicule depuis ses débuts un éthos apparemment très éloigné de la mort (jeunesse, amour, insouciance, audace, énergie, modernité, rapidité, expérimentation) qui néanmoins, par la prise de risques que ces différents ingrédients impliquent, cohabite en permanence avec la possibilité (voire l'espoir) d'une mort prématurée. Un des leitmotivs du rock est bien le souhait qu'exprime le protagoniste de «My Generation» (The Who, 1965): «I hope to die before I get old ».

\section{Mort et immortalité}

Réfléchir à la façon dont la critique a commenté l'ultime album de David Bowie et la disparition de son auteur m'a semblé d'autant plus pertinent que Bowie a souvent été comparé au personnage de Dorian Gray créé par Oscar Wilde, à son obsession pour la jeunesse et l'immortalité et à son hédonisme sans limites ${ }^{1}$. Ainsi, Ana Leorne observe :

The obsession with youth and immortality was a constant in Bowie's early career, and the fact that he himself gave up a part of his own life-his personal side-to be immortalised through Art (his own), makes him the perfect example of someone affected by Dorian Gray syndrome ${ }^{2}$ as he was seeking, in his own way, immortality. (Leorne 2015, 113)

La presse a souvent eu recours au parallèle entre Gray et Bowie. Thomas Burgel évoque «cet infini Dorian Gray» (Burgel 2016, 16), tandis que dans un article au titre explicite ( ( David Bowie, le Dorian Gray du rock ») Stéphane Davet commente ainsi la campagne publicitaire «Chaque jour une vie nouvelle » pour Vittel dans laquelle Bowie apparaît sous les traits de ses différents avatars :

On comprend que les publicitaires d'une grande marque d'eau minérale aient pensé à lui. C'est ainsi qu'on le voit le temps d'un écran de publicité [...] suggérer les vertus rajeunissantes de leur produit. En bande-son du spot, le Dorian Gray du rock proclame: "Je ne vieillirai jamais." Il s'agit d'un extrait d'un morceau, "Never Get old", qui figure également sur son nouvel album, Reality, le vingt-sixième de sa discographie en studio. (Davet 2003)

11 Le décès de Bowie a donné à la presse l'occasion de revenir à cette comparaison, sous laquelle transparait cette fois une pointe d'ironie, de déception ou d'incrédulité. Stéphane Koechlin en fait le titre de sa nécrologie pour Valeurs Actuelles, « David Bowie, mort du Dorian Gray de la musique pop» (Koechlin 2016). Dans Le Monde, Auréliano Tonnet écrit : «De même que Dorian Gray ne vieillissait qu'en peinture, David Bowie 
n'a vieilli qu'en voix» (Tonnet 2016). Daniel Salvatore Schiffer amorce dans un article titré «Mort d'un dandy stellaire » une réflexion qu'il a développée dans un ouvrage publié en mai de la même année : «David Bowie, que l'on aurait cru éternel tant ce Dorian Gray de la musique semblait ne pas vieillir, dépasser les âges, transcender les modes et réinventer les styles, est donc mort " (Schiffer 2016). Schiffer cite ensuite plusieurs extraits du roman qui démontrent la proximité entre Bowie et Gray, une volonté comparable de jouir de l'instant présent et de profiter de la jeunesse face aux ravages du temps, en particulier le passage suivant :

Car il est si bref, le temps que durera votre jeunesse [...] Nos membres nous font défaut, nos sens se décomposent. Nous dégénérons, et devenons des pantins hideux [...] Il n'y a absolument rien en ce monde que la jeunesse ! (Schiffer 2016)

David Bowie lui-même a joué avec cette réputation. La vidéo promotionnelle du titre «Look Back in Anger" que réalise David Mallet en 1979 nous montre un Bowie désespéré finissant de peindre son autoportrait en ange coruscant tandis que son propre visage de décompose sous ses yeux dans un miroir.

Contrairement à David Bowie, la plupart des rock stars de sa génération n'ont pas voulu (ou pas pu) cacher les atteintes de l'âge. Certains ont même choisi de forcer le trait et d'afficher au grand jour ce que d'autres cherchent à faire oublier. Avec un certain courage (à moins qu'il ne s'agisse d'un ego surdimensionné), des artistes comme Iggy Pop ou Keith Richards exhibent leur corps, affichent sans vergogne leurs stigmates, dévoilent la maigreur et la cicatrice, la «blessure » dont parle Foucault (Foucault, 75), refusant ainsi avec insolence les impératifs esthétiques de notre époque, qui voudraient que l'on cache les dégâts les plus visibles et les outrages les plus infamants. Aux puissances médiatiques qui exigent lifting et Photoshop, ils opposent une indépendance qui passe, comme au temps de leur jeunesse, par l'affranchissement des codes sociaux et esthétiques en vigueur. Après avoir, au cours des années soixante, contribué à la fétichisation de la jeunesse, ces artistes imposent aujourd'hui leurs rides et leurs flétrissures comme un nouveau triomphe, une nouvelle victoire. Aux critiques goguenards, ils répondent par l'indifférence et le dédain et affirment leur mépris des lois qui régissent le comportement dans l'espace public. En s'affichant dans leur décrépitude dérangeante, ils modifient les codes de la représentation et posent les bases de nouvelles modalités d'être. En cela, ils s'affirment aujourd'hui encore, malgré l'argent, la gloire et diverses formes de canonisation, comme véritablement dissidents au sein d'une société obsédée par l'apparence et la perfection formelle au nom d'une transparence et d'une « authenticité » présentées en valeurs ultimes.

L'attitude de ces artistes rejoint peut-être à sa façon l'ambition de David Bowie, celle de pouvoir durer, jusqu'au bout de la vie et plus loin encore, malgré le corps, et au-delà du corps. Mais la plupart des stars vieillissantes, au lieu de porter cette ambition comme Bowie en triomphant de façon surnaturelle des atteintes de l'âge, lui confèrent une certaine humanité en acceptant avec humilité et lucidité les réalités physiques. Elles offrent, à contre-courant de l'insistance technologique "post-humaine » contemporaine, présentée comme la nouvelle frontière, une acceptation de la fragilité et de la finitude de l'être humain.

Or, après avoir longtemps esquivé cette humanité, David Bowie choisit lui aussi, dans Blackstar, d'exhiber les traces du vieillissement, ou plus précisément de la maladie. Plutôt que de les dissimuler comme Freddy Mercury l'avait fait dans ses ultimes vidéos ${ }^{3}$, Bowie surenchérit sur l'ambiance mortifère, multipliant les allégories textuelles et 
visuelles qui seront décryptées après sa mort comme autant d'allusions à son départ imminent. Certes, il ne s'agit peut-être pas là d'une monstration apaisée de la "réalité " et ces mots et ces images ne sont peut-être qu'une mise en scène de plus, un dernier masque, un ultime avatar. Mais la rupture avec les choix esthétiques antérieurs est nette.

À l'instar du «Modern Love» (1983) que décrivait Bowie, peut-on alors parler de sa mort comme d'une "modern death » ? Son décès est le premier d'une star de rock de ce calibre à être mis en scène avec autant de lucidité, le premier à faire l'objet d'une telle organisation, d'une telle anticipation artistique et esthétique par le futur défunt, le seul à être immédiatement précédé d'un album apprécié non pas parce qu'il est son dernier mais parce qu'il s'agit, pour la majorité des critiques et du public, d'une œuvre majeure, novatrice, exploratoire. A-t-il pour autant donné lieu à des commentaires proprement inouïs, à un nouveau regard sur la mort, à un nouveau rapport à cet événement ? Constitue-t-il une mort rock, ce que n'ont pas été les décès, inopinés ou attendus, mais non préparés de Jimi Hendrix, Janis Joplin, Jim Morrison, Brian Jones, John Lennon, Kurt Cobain, John Bonham, Ian Curtis, Marc Bolan, Bob Marley, Keith Moon, Elvis Presley, Frank Zappa, George Harrison, Amy Winehouse, Michael Jackson, Sid Vicious ? A-t-on parlé de l'œuvre de Bowie, de ce dernier opus en particulier, en des termes différents, spécifiques à cette mise en scène visant à se démarquer, une ultime fois, des autres mortels, fussent-ils l'aristocratie du rock?

\section{Louanges et critiques}

17 Les critiques de Black Star et des vidéos qui l'accompagnent offrent des différences notables selon qu'il s'agit de la presse française ou anglophone et qu'elles ont été rédigées avant ou après l'annonce de la mort de David Bowie (certains avouent d'ailleurs avoir refait leur texte en fonction de la nouvelle). Dans la presse francophone, les premières critiques sorties avant la parution de l'album ou le jour même de sa sortie révèlent des avis partagés. Fabrice Pliskin a des mots assez durs. Il trouve l'album " un peu lassant ", estime que ses sonorités drum and bass n'apportent « rien de nouveau » (Pliskin 2016). Certaines mélodies sont «assez fades», la nouvelle transformation de Bowie est «à moitié convaincante ", plusieurs morceaux se perdant «dans les sables de la banalité ». Sylvain Siclier trouve que «la plupart des compositions semblent durer trop longtemps » (Siclier 2016), opinion que partage Hugo Cassavetti qui estime qu'« il reste à voir » si l'album est une réussite (Cassavetti 2016). C'est le saxophone de Donny McCaslin, affilié à l'avant-garde new-yorkaise, qui s'attire le plus de critiques: "envahissant" (Olivier Nunc 2016), "guère renversant" (Siclier 2016), "très (trop ?) présent» (Cassavetti 2016), " passe-partout, tendance variété française des années 1980 » (Pliskin 2016).

D'autres chroniqueurs sont au contraire enthousiasmés dès la première écoute. Olivier Cachin nous avertit: «Attention, ovni », et il accumule les termes élogieux : «le choc du single ", " entre le grandiose et l'intime, l'avant-garde et la pop cintrée », le disque d'un artiste «qui n'a jamais été aussi loin dans l'inconnu » (Cachin 2016). Il conclut sa critique en affirmant qu'il s'agit d'une œuvre d'une «émancipation totale, la liberté free » et que "cette étoile brillera dans la nuit». De façon générale, c'est le côté expérimental de l'album qui est salué, son originalité radicale, la capacité de Bowie à se remettre en question, à explorer d'autres territoires que le rock. Louis Nadeau parle 
d'« un nouveau pic de créativité » (Nadeau 2016), Rebecca Manzoni, au même titre que Richard Philippe ou Antoine Guillot, affirme qu'on est « loin du rock» (Manzoni 2016). Cachin précise que l'album est anti-rock, « ou plutôt anti-pop ", par quoi il doit vouloir dire anti-commercial ${ }^{4}$. C'est d'ailleurs un aspect qui revient sous la plume de plusieurs journalistes, le plus souvent comme un compliment : l'album est complexe, exigeant, difficile d'accès, il s'adresse à une élite éclairée, ne comporte pas de tube (Manzoni 2016), et en conséquence il aura du mal à passer en radio (Nadeau 2016) et à «briller dans les charts » (Cachin 2016). Certains s'interrogent également, du fait même de cette esthétique novatrice et exigeante, sur sa pérennité, sa capacité à durer (Cassavetti 2016). Enfin, l'ensemble des commentaires souligne son ambiance élégiaque. Nadeau parle de "pessimisme crépusculaire», LCI de "ballade crépusculaire» (LCI 2016), Cassavetti de "renaissance dans l'obscurité » et Richard de «tonalité élégiaque » (Richard 2016).

Le ton change radicalement pour les commentaires publiés quelques jours après la sortie de l'album, une fois la mort de David Bowie connue. On y retrouve les allusions à la spécificité de l'album, à son ambiance "mortifère» (Schiffer 2016) ou "crépusculaire» (Huffpost 2016), à sa difficulté d'accès ("Pas le genre de musique à mettre entre toutes les oreilles»), justifiée parfois non par son originalité intrinsèque, mais par le fait qu'il s'agit du dernier album publié du vivant de l'artiste. En revanche, il devient difficile de trouver une critique négative, exprimant réserve, doute ou incompréhension. Pour les amateurs comme pour les professionnels, l'album est « une tuerie absolue", "son meilleur depuis Heroes", " enivrant, puissant, incroyable et désormais éternel »(Clash 2016).

Par ailleurs, de nouveaux qualificatifs apparaissent : «testament » (Huffpost, Schiffer, Péron et Gester, Viviant, Sébastien, 2016), «message d'adieu » ou "cadeau d'adieu » (Sébastien 2016), qui soulignent la volonté lucide de Bowie de clore sa carrière et son œuvre en toute conscience, en livrant à son public un ouvrage expressément conçu pour être son ultime expression musicale. En revanche, si ces qualificatifs constituent un leitmotiv facile et prévisible des critiques de Blackstar, peu de journalistes se risquent à définir précisément le contenu de ce testament. Une voix conteste cette lecture, une seule, d'autant plus forte qu'elle est celle du principal musicien de l'album, le saxophoniste Donny McCaslin. Répondant, un an après la mort de Bowie, à une question portant sur l'aspect «testamentaire » de l'album, il répond : «J'ai lu tant de choses là-dessus. Mais d'après moi, il allait de l'avant, parlait d'enregistrer davantage de musique. Ce simple fait me laisse à penser qu'il ne s'agit pas d'un adieu » (AFP, David Bowie 2016).

21 La presse anglophone, britannique comme étatsunienne, a encensé l'album dès la parution des premiers singles de façon beaucoup plus systématique que son homologue française. Il est presque impossible de relever la moindre réserve. Les termes utilisés sont systématiquement élogieux, les superlatifs abondent : «the most extreme album of [Bowie's] entire career » (Andy Gill 2016) », « extraordinary » (Neil McCormick 2016), "a defining statement" (Michael Rancic 2016), "singular, unique sound, trippy, majestic, breathtaking, relentlessly fascinating, a stunning return» (Chris Gerard 2016), «a masterpiece» (Fricke 2016), «intoxicating» (Vishnevetsky 2016). Vishnevetsky ajoute une phrase qui résume la tonalité générale des recensions: "Bowie is now back to making music as art» (Vishnevetsky 2016). De façon symptomatique, les interventions au saxophone de McCaslin que la presse française 
boudait sont unanimement saluées par les Anglo-Saxons, à l'instar de Chris Gerard (qui les qualifie de "phénoménales", Gerard 2016). Les avis plus nuancés des Français seraient-ils dus à une moins grande familiarité avec le jazz d'avant-garde et les musiques improvisées (puisqu'il s'agit-là de l'univers musical dans lequel évolue McCaslin), ce qui les rendrait moins à même de les apprécier ou au contraire capables d'une plus grande distance critique ?

Les critiques anglophones rejoignent en revanche les français quant à la dimension expérimentale de Black Star: "one of the most aggressively experimental records the singer has ever made » (Fricke 2016), " This is why the world still needs David Bowiefor the unexpected» (Gerard 2016), "a reasonable idea of what to expect - i.e., the unexpected " (Richards 2016), "venturing once more into the outer limits of pop " (McCormick 2016), « a slate-cleaning break with the past » (Petridis 2016), « a rejection of pop's prissy little contours» (Harman 2016). Embling est le seul à oser décrire l'album dans les termes suivants: "an unsentimental collection of pop songs ». Ils soulignent également la prise de risque commerciale: "There are no potential hits here » (Gerard 2016), «It is unlikely to worry Adele about her prospects for dominance of the charts» (McCormick 2016), "Bowie isn't uncumbered with commercial aspirations » (Allmusic 2016).

Une fois la mort de l'artiste rendue publique, comme en France, de nouveaux termes apparaissent, faisant de Blackstar «a farewell " (Griggs 2016), "a final message » (Coscarelli \& Paulson 2016), «a parting gift 》 (Gerard 2016), « a final statement » (The Guardian 2016), «epitaph» (Harman 2016), «eulogy» (Crockford 2016), «a heartbreakingly sad way to bid farewell» (Cooper 2016). "Testament» ne fait néanmoins pas partie du vocabulaire de référence anglophone.

\section{Une rhétorique de l'oraison funèbre}

En revanche, au-delà d'un contenu remarquablement homogène, la façon dont sont construites et rédigées les critiques francophones et anglophones post-mortem de Blackstar présente des différences notoires. Les articles en français font preuve d'une surprenante uniformité dans leur agencement rhétorique. On y observe tout d'abord une organisation temporelle systématique : des remarques sur le présent (le décès de Bowie, le disque qui vient de sortir et l'émotion que suscitent ces deux événements) ; un retour sur les accomplissements et l'œuvre de Bowie, souvent sur le mode hérö̈que ; enfin, quelques réflexions sur la place que l'album et, de façon plus générale, l'œuvre de Bowie sont destinés à occuper dans le futur. Péron et Gester parlent ainsi dans un premier temps de "stupéfaction" à l'annonce du décès de Bowie (Péron et Gester 2016), avant d'entamer une seconde partie biographique, qui débute avec le rétrospectif "tout au long de sa carrière", puis de revenir plus en détail sur sa vie privée et artistique à partir de "Il est né le 8 janvier 1947 ». Langs, malgré sa distance et son ironie, reconnaît que «La mort de David Bowie est un séisme tel qu'on en connaît rarement» (Langs 2016). Clash clôt sa chronique avec un «on ne t'oubliera jamais » (Clash 2016), tandis que Jean-Marie Pottier imagine quelle sera la réponse au questionnaire de Proust dans vingt ans : « En 2036, nul doute que quelqu'un répondra David Bowie " (Potier 2016) et Auréliano Tonet en s'intéressant au futur des cordes vocales de Bowie, assurant que « d'album en album, malgré le travail du temps, elles garderont cet alliage d'élasticité et de tension, de flammes et de cendres, de théâtralité 
et de sincérité » (Tonet 2016). Et Langs renchérit : « plus rien ne sera vraiment pareil, et le simple fait de le dire est une inquiétude pour l'avenir » (Langs 2016).

Cette organisation, peu surprenante en soi et néanmoins propre aux commentaires en français, correspond point par point aux éléments définitoires de l'oraison funèbre dans la forme prise par celle-ci en France à partir du $16^{\mathrm{e}}$ siècle et surtout au $17^{\mathrm{e}}$ grâce en particulier dans les textes de Bossuet ${ }^{5}$, au point que l'on peut envisager l'ensemble des critiques publiées en France comme des formes contemporaines d'oraison. Christine Noille définit ainsi deux « canevas » principaux d'oraisons, les " pauvres », ou "disqualifiées", et les "valorisées", selon leur degré de complexité. Au sujet des premiers, elle écrit ceci, dont la correspondance avec la structure des critiques est frappante :

Le canevas disqualifié est identifié par les trois temps verbaux : il traite d'abord des choses présentes (l'état présent du défunt, selon une modalité fortement pathétique), puis des choses passées (narration du caractère et des actions remarquables du défunt, sur le mode de l'éloge), enfin des choses futures (recension des espérances définitivement déçues, afin d'émouvoir. (Noille 2015, 42)

Noille insiste en outre sur la nature hybride de l'oraison, "genre du discours au carrefour de l'éloge, de la lamentation, de la consolation et de l'exhortation » (Noille 2015, 42), autant d'éléments qui correspondent à la tonalité plurielle des critiques françaises. De fait, deux thématiques centrales s'en détachent. La première est l'expression d'une profonde tristesse, partagée par des millions de fans, qui plus encore que les professionnels, déclinent leurs commentaires sur le triple mode du chagrin que leur occasionne le décès de la star, du réconfort qu'ils se prodiguent les uns aux autres et de leur espérance de dépasser la douleur. Jean-Marie Pottier confie: "Tous, ou presque, nous avons pleuré la mort de David Bowie. Nous avons tweeté, facebooké, instagrammé, étalé sur tous les réseaux sociaux notre peine sincère ». Langs affirme «la peine est un sentiment universel» (Langs 2016) et Clash se lamente qu'il ne peut "rien faire d'autre que pleurer comme un bébé ", ajoutant avec la crudité propre aux blogs de fans : " putain, ça fait mal d'écrire ça » (Clash 2016). Nous sommes bien ici dans une forme de lamentation conforme aux modèles qu'ont établis les Anciens ${ }^{6}$.

Seconde thématique à émerger et qui rejoint la typologie définie par Noille, l'éloge, d'autant plus évident que les remarques négatives disparaissent presque totalement des critiques publiées après sa mort. On peut lire le désir de rappeler, une ultime fois, avec force superlatifs, l'incroyable parcours de l'artiste, son originalité, sa beauté, ses prouesses, sa capacité à nous surprendre, nous séduire, nous exciter, nous émouvoir et la marque indélébile qu'il a laissée sur son époque et sa société par sa mise en scène de l'androgynie et de la bisexualité. Or, comme le rappelle Sophie Hache, l'une des fonctions de l'oraison est bien évidemment de faire l'éloge du défunt en rappelant « les éléments majeurs de sa vie» (Hache 2009) et de susciter une émotion collective en ayant recours à diverses formes d'amplification, c'est-à-dire "une augmentation du matériel lexical et phrastique » (Molinié 92), en particulier grâce à l'hyperbole « dont l'adjectif, associé à l'expression du comparatif et du superlatif, est précisément un support privilégié » (Hache 2014). Ces adjectifs foisonnent dans les critiques de Black Star, sous diverses formes, chacune étant représentative de l'amplification propre à l'oraison telle que Hache l'a définie : oxymores («l'œuvre entière, grandiose y compris dans sa fragilité ", " entre le grandiose et l'intime ", "funk glacé ", " élégant chaos ", "douceur inquiète») ; clichés et stéréotypes ("intuition foudroyante», "chansons intemporelles », "génie torturé», « envolées lyriques»); antithèses («j'étais 
convaincu qu'il mourrait jeune, à l'issue d'une stupéfiante carrière ", « il entretient le flou sur son identité, reste insaisissable, indéfinissable, bien que sa patte demeure reconnaissable entre mille »).

Malgré les louanges post-mortem déversées sur Black Star, la critique française ne manque pas d'égratigner la figure de Bowie. À l'instar de Péron et Gester qui rappellent les « décennies d'errances douloureuses (les navrantes années 80 en tête) » (Péron et Gester 2016), elle évoque les albums ratés, les excès de substances diverses, la vanité du personnage, sa tendance à suivre les modes plus qu'à les créer, à être là où il faut, quand il le faut, tel un simple caméléon surdoué. L'oraison funèbre étant de nature hagiographique, ces quelques admonestations et dénigrements n'invalident-ils pas notre hypothèse d'une correspondance entre les critiques de Blackstar et le mode de l'oraison ? Au contraire, Hache insiste sur une composante majeure de l'oraison, soulignée par les théoriciens du $18^{\mathrm{e}}$ siècle, qui consiste à concilier, à des fins de crédibilité accrue, louanges et modération, raison et passion, de conjuguer l'admiration, forcément subjective, avec un sens nettement plus objectif de la mesure. Il reviendrait donc de suivre l'exemple de Massillon qui «se dégage du soupçon de mensonge brillant attaché à l'oraison funèbre, en accordant une place à la critique » (Hache 2009). Hache cite ainsi Paul Pellisson-Fontanier :

Il faut louer le Roy par tout, mais pour ainsi dire sans louange, par un récit de tout ce qu'on lui a vu faire, dire \& penser, qui paroisse désintéressé, mais qui soit vif, piquant, \& soutenu évitant dans les expressions tout ce qui se tourne vers le Panegyrique. Pour en être mieux cru, il ne s'agit pas de lui donner là les épithètes \& les éloges magnifiques qu'il mérite : il faut les arracher de la bouche du Lecteur par les choses mêmes. (Pellisson-Fontanier 1735, 326)

Il suffirait de remplacer le Roy par Bowie pour avoir une description fidèle des commentaires rédigés par la critique française en l'an de grâce 2016.

Les parallèles entre les recensions françaises de Black Star et l'art de l'oraison tel qu'il se pratiquait en France aux $16^{\mathrm{e}}$ et $17^{\mathrm{e}}$ siècles ne s'arrêtent pas là. Un trait récurrent des commentaires est la liste qui est dressée des nombreuses récompenses obtenues par Bowie (awards, chiffres de vente, classement dans les charts, etc.). Cicéron lui-même recommandait d'inclure dans les oraisons de tels rappels : "Les honneurs décernés avec éclat, les prix accordés au mérite, donnent aussi beaucoup de lustre aux éloges » (Cicéron 1821, 451). En outre, de nombreux critiques insistent, à des fins de consolation, sur une rémanence bowienne, non seulement à travers son œuvre, mais via le rapport qu'il continuerait d'entretenir, de l'au-delà, avec son public. Les paroles de plusieurs chansons de l'album sont convoquées pour confirmer l'éventualité de cette relation spirituelle: "something happened on the day he died, spirit rose» ("Blackstar»), «Look up here, I'm in heaven " ( Lazarus »), « don't believe for just one second I'm forgetting you " ( Dollar Days»), «seeing more and feeling less» («I Can’t Give Everything Away »). Développant les allusions religieuses, les critiques suggèrent des rapprochements avec le Christ de Mantegna, avec "Lazare ressuscité d'entre les morts » (Tonet 2016). Or, si l'oraison funèbre demeure chez les Anciens « étrangère à la pensée d'une autre vie, à la perspective consolante d'un bonheur éternel » (Jodra, Imago Mundi), la pensée chrétienne exige que l'oraison soit le moment d'un rappel de la permanence de la vie dans l'au-delà, de l'existence d'une organisation dont le paradis évoqué par Bowie et les critiques fait partie. Au-delà de la simple métaphore désémantisée, le « heaven » de Bowie a bien été perçu par la critique française comme 
une allusion non seulement spirituelle, mais aussi bel et bien chrétienne, à une vie après la mort telle qu'elle est conçue dans une oraison funèbre.

\section{Une rhétorique de la consolation}

31 En revanche, aucune des modalités rhétoriques si présentes dans la presse française (construction temporelle, amplification, références chrétiennes, distance critique) ne se retrouve dans les critiques anglophones publiées après le décès de David Bowie. C'est une autre forme, elle aussi très codifiée, qu'on peut y déceler.

L'essentiel de la critique anglophone s'articule autour de quelques traits rhétoriques récurrents. Le plus frappant est l'usage systématique d'une première personne du pluriel à portée inclusive pour évoquer l'impact émotionnel de la mort de David Bowie et de son dernier album : « How David Bowie told us he was dying », « He made Blackstar for us » (Cooper 2016), « Bowie kept us mesmerized » (Payne 2016), « he will always be with us » (Vincent 2016), «we're still obsessing over David Bowie's death » (Rogers 2016), « forcing us to think of him in the past tense » (O'Neal 2016), « we'd already kind of said goodbye to him once before, we will never, sadly, get to hear the next chapter " (Rayner 2016), "we will get to see him reborn countless times over the coming decades » (Jonze 2016), «I have to put Bowie in the past tense now, as we all will have to » (Crockford 2016), «We all know that Blackstar is an album [that] Bowie wanted us to engage with» (Mathers 2016), "death is scarry, and we're all staring it down » (Embling 2016), «we are millions» (Duldig 2016), «We all work through grief in our own ways» (Tabakis 2016), "the inevitable long goodnight that awaits us all» (Thompson 2016). On observe dans ce cas une volonté patente non seulement de s'inclure dans une communauté de tristesse, mais aussi de prendre en compte le chagrin des autres pour faire advenir cette communauté, de lui donner corps, avec l'impact consolatoire qu'une telle inclusion peut avoir. Autre modalité de la mise en place de cette communauté, la fréquence des citations de proches de Bowie, notamment de ceux qui ont travaillé sur ses ultimes projets: James C. Nicola, le directeur du New York Theater Workshop où fut jouée la pièce Lazarus, Ivo van Hove, son directeur musical, Robert Fox, le producteur, les musiciens du groupe, Brian Eno, Johan Renck, le réalisateur des vidéos. Tous contribuent, en partageant leur chagrin, à actualiser l'hyperbole de Vincent : « the world mourned Bowie » (Vincent 2016).

Une fois l'empathie avec le public instaurée, les journalistes insistent sur la nécessité de ne pas céder au désespoir, d'avoir confiance en une vie possible après - et sans - David Bowie, nécessité rendue envisageable grâce à la visée expérimentale et créative du dernier album. Chris Payne en fait «an open-ended elegy, one that urges us to experiment onward » (Payne 2016), Sean O'Neal insiste sur le fait qu'il ne s'agit pas d'un "funeral dirge ", mais plutôt d'une "resurrection, designed to be puzzled over for years» (O'Neal 2016), Jude Rogers, paraphrasant un morceau de 1. Outside, écrit de l'album qu'il constitue « a new career in a new town, in the stars, everywhere » (Rogers 2016). Pour Jonze, « we can be sure of something : as Bowie's influence stretches far and wide, seeping into the work of those whose lives he touched, we will get to see him reborn countless times over the coming decades " (Jonze 2016). O'Neal donne un aperçu de la tonalité dominante de la presse anglophone par cette remarque finale: «David Bowie may be dead, but that doesn't mean he can't still surprise us » (O'Neal 2016). C'est de fait avec leur dernière phrase que la plupart des critiques profèrent à leurs 
lecteurs (et à eux-mêmes) leurs plus fortes exhortations : " his gift to humanity will never die » (Duldig 2016), « He will haunt us for decades to come » (Watson), «we can only hope it isn't the end of a career » (Mathers 2016).

Claudie Martin-Ulrich nous rappelle en ouverture d'un numéro spécial d'Exercices de rhétorique consacré à la consolation que "notre besoin de consolation est insatiable » (Martin-Ulrich 2017, 1). Cette maxime, qu'elle emprunte à Stig Dagerman (1981), permet de mettre en perspective les différentes remarques qui précèdent. De fait, si la critique française a puisé dans le modèle de l'oraison, de façon probablement inconsciente, de quoi rendre compte de l'ultime album de David Bowie, c'est de toute évidence le mode de la consolation que les anglophones ont adopté. Car si, comme le précise Martin-Ulrich, « on pense couramment que, produite par un élan du cœur, la consolation est spontanée donc désordonnée, ou du moins sans forme préétablie possible, sans plan ni patron " (Martin-Ulrich 2017, 1), cette forme essentiellement littéraire, épistolaire même, dont le but est d'«apaiser la douleur, éradiquer la mélancolie, soulager la peine par des mots » (Martin-Ulrich 2017, 2) suit généralement un modèle rigoureux en deux temps : il s'agit tout d'abord de créer de l'empathie avec celui qui souffre, de lui faire comprendre qu'on partage sa peine, que l'on éprouve un chagrin similaire, ce à quoi s'emploient les différentes techniques d'inclusion communautaire observées précédemment. Mais cette empathie n'est pas gratuite. Elle vise à rendre possible la fonction principale de la consolation, une exhortation à se reprendre : « le consolateur dialogue avec son interlocuteur affligé, se rapproche de lui tout d'abord pour mieux, dans un second mouvement, lui faire la leçon et l'entraîner sur son propre terrain, celui de la raison » (Martin-Ulrich 2017, 5), c'est-à-dire, in fine, l'amener à retrouver sa place dans le concert humain, dans la société des vivants que la douleur nous a fait quitter un instant, société des affligés dans un premier temps, puis société tout court. Là encore, les nombreuses exhortations que prodigue la presse anglophone lorsqu'elle nous incite à considérer l'avenir avec confiance en nous appuyant sur le modèle de créativité et d'expérimentation que Bowie nous a proposé avec Black Star, correspondent exactement au second mouvement de la consolation. Plus encore, elles soulignent quelles sont les valeurs considérées comme les plus nobles aujourd'hui dans notre société, en l'occurrence les valeurs d'originalité et d'inventivité, corroborant ainsi les propos de Martin-Ulrich et confirmant que nous sommes bien en présence du mode consolatif :

La consolation est un observatoire très éclairant pour appréhender les valeurs d'une société donnée [...] Elle permet de saisir les spécificités historiques et éthiques d'une époque, ses aspirations et ses doutes [...] Les discours sur la consolation sont des indices précieux de la façon dont une société appréhende les liens entre les individus, dont elle éduque à bien vivre, protège, se défend et corrige. (Martin-Ulrich 2017, 3-8)

Reste qu'au-delà des intentions du consolateur, l'impact de ses efforts sur l'endeuillé reste difficile à apprécier et qu'il ne nous est guère possible d'effectuer, comme Paula Barros nous y engage, "une réflexion sur la question de la réception de ce genre discours » et de «la prise en charge de la douleur d'autrui » (Barros 2017, 2) pour en évaluer les vertus et les limites. Nous pouvons en revanche revenir sur l'adéquation de cette tradition consolatoire à l'événement saisissant auquel la presse anglophone et francophone l'a appliquée. 


\section{Une mort rock ?}

\section{l'oraison et de la consolation, à la lumière des formes rhétoriques éprouvées auxquelles} elle a eu recours, est-elle en mesure de faire saisir la radicalité et la modernité du geste de David Bowie ? Rend-elle compte avec justesse de sa démarche inouïe qui a consisté à contrôler jusqu'au bout un processus créatif lui permettant de façonner un écrin rock pour clore son passage sur terre et parachever son œuvre ? A-t-on saisi, en la lisant, qu'il s'agissait d'une "modern death » tout aussi révolutionnaire que les différentes formes de "modern love » que Bowie chantait ? A-t-elle rendu justice à la dernière phrase de «Lazarus » : «Oh I'll be free, ain't that just like me »?

De toute évidence, la presse n'est pas libre, pas plus que les fans. De toute évidence, sans surprise, elle est grégaire et mimétique. À de rares exceptions près (Langs, Embling), elle cite les mêmes références, fait allusion aux mêmes faits, se repaît des mêmes anecdotes, utilise les mêmes modalités rhétoriques. Malgré elle, et grâce à elle, nos deuils, comme l'écrit Jean-Marie Pottier, sont devenus consensuels, « rassemblant tout le monde au garde-à-vous devant le monument, du grand-père qui ne s'est jamais remis du 'Starman' de 'Top of the Pops' à l'ado qui a écouté Blackstar pour la première fois sur Spotify ou YouTube » (Pottier 2016). Pour souligner le chemin parcouru par la critique et par la société qu'elle représente dans la voie d'un consensus timoré et pusillanime, qui dénature l'art - le rock - de ses vertus, Pottier cite les commentaires rédigés dans Rock'n'Folk par Philippe Paringaux en novembre 1970 à l'occasion de la mort de Jimi Hendrix :

Pour le camp principal, c'étaient des drogués et des crados et des fous faiseurs de bruit. Des corrupteurs qui, après avoir donné toute leur vie le mauvais exemple, en donnent enfin un bon en s'en allant rapidement, en crevant comme des chiens dans leurs vomissures. [...] La jeunesse, pourtant, a montré en la circonstance un peu plus de dignité, peut-être parce qu'elle seule contre le reste du monde - c'est-àdire qu'elle n'a aucun pouvoir sur les moyens d'information. (Paringaux 1970)

S'il a pu se travestir en Diamond Dog, Bowie n'a pas crevé comme un chien dans sa vomissure. Et les jeunes, ou ceux qui l'ont été, n'ont guère fait preuve de dignité car ce sont eux qui, aujourd'hui, contrôlent les moyens d'information. Bowie a été embaumé par des rhétoriques archaïques à l'instant même de sa mort, sur les blogs de fans tout autant que par les professionnels. Effectivement, oraison et consolation sont des modes très révélateurs du fonctionnement et des valeurs de nos sociétés. Ils nous disent à quel point, tandis que David Bowie a continué à se remettre en question jusqu'à sa dernière création, jusqu'à faire de sa mort sa dernière création, la critique (et nous avec, n'ayons pas d'illusions), avons au contraire recherché la sécurité, n'acceptant du risque et du danger, fussent-ils esthétiques, que ce que nous en offre par procuration l'artiste.

$\mathrm{Au}$ bout du compte, la rhétorique de la consolation et les stratégies propres à l'oraison, en insistant sur l'importance de la raison et de la mesure dans l'expression de la douleur, participent de ce que Paula Barros appelle «la réaffirmation commune du souci de l'autre comme fondement éthique de la vie en société » (Barros 2017, 11). Touchés par la même peine, atteints par la même violence, lecteurs et critiques ont été puiser dans un patrimoine commun pour s'apporter soutien et réconfort, resserrer le lien social, et redire, au-delà des excès et de l'anticonformisme communément associés 
à l'univers du rock, les vertus «de la retenue émotionnelle en mettant en avant les valeurs de rationalité, de masculinité et de sagesse » (Barros 2017, 11).

C'est une vieille mort, des plus classiques, des plus traditionnelles, compassée, prévisible, un enterrement de première classe que nous avons offerts à Bowie, lui qui avait choisi de nous bousculer jusqu'au dernier moment dans nos certitudes et nos conforts. Du rock, de la modernité du rock telle qu'il l'a redéfinie jusqu'au bout, il n'est rien resté. Ce qui subsiste, aujourd'hui comme il y a deux mille ans, c'est une telle frayeur face à la mort que seuls les réflexes les plus codifiés, les plus éprouvés surgissent pour l'apaiser. Respect transi, canonisation, sanctification, nous n'avons rien trouvé de mieux. Pour preuve, l'exposition pas même ironique intitulée "Saint Bowie » qu'organisait la galerie Stephen Roman de Brooklyn du 2 mars au $1^{\text {er }}$ mai 2016 et qui rassemblait «des reliquaires, des ex-votos, des autels, des photos et autres objets à fonction magique destinés à communier avec Bowie dans l'au-delà » (Shishigami 2016), transformant Bowie en un « digital bodhisattva » (Powers 2016).

Il ne manque plus qu'un «tombeau », ce genre musical très prisé à la période baroque, pour parfaire la canonisation poussiéreuse, un tribute album de plus enregistré par quelques stars compassées qui viendront enfoncer le dernier clou sur le cercueil de Bowie, et sur celui du rock par la même occasion. Mais peut-être, après tout, cette canonisation n'est-elle pas si étrangère, si antinomique au rock. L'adoration d'une star, fût-elle noire, ne constitue-t-elle pas, via le principe sacrificiel, l'essence même du rock ${ }^{7}$ ?

\section{BIBLIOGRAPHIE}

\section{Sources primaires}

«Alors il vaut quoi ‘Blackstar', le dernier album de David Bowie ? ». LCI, 8 janvier 2016. Consulté le 15 juin 2017. <http://www.lci.fr/musique/alors-il-vaut-quoi-blackstar-le-nouvel-album-dedavid-bowie-1500376.html>

AFP. « 'Blackstar' : un album sombre et surprenant en guise de testament pour David Bowie ». Huffpost, 11 janvier 2016. Consulté le 15 juin 2017. <http://quebec.huffingtonpost.ca/2016/01/11/ david-bowie-blackstar-un-album-sombre-et-surprenant-en-guise-de-testament-

_n_8953290.html>

AFP. « David Bowie : sa dernière audace racontée par un collaborateur ». Le Figaro, 3 janvier 2017. Consulté le 15 juin 2017. <http://www.lefigaro.fr/musique/

2017/01/03/03006-20170103ARTFIG00237-david-bowie-sa-derniere-audace-racontee-par-uncollaborateur.php>

Allmusic. « Blackstar ». Allmusic. Consulté le 15 juin 2017. <www.allmusic.com/album/blackstarmw0002894417>

Bowie, David. Blackstar. ISO, RCA, Columbia, Sony, 2016.

Bowie, David. Look Back in Anger. dir. David Mallet. RCA. 1979. 
Britton, Luke Morgan. « Did David Bowie name 'Blackstar' album after his own cancer lesion? ». NME, January 13, 2016. Consulté le 15 juin 2017. <http://www.nme.com/news/music/davidbowie-129-1194972>

Burgel, Thomas. « David Bowie : clip effrayant, bizarre et beau pour 'Lazarus' « . Les Inrocks, 7 janvier 2016. Consulté le 15 juin 2017. http://www.lesinrocks.com/inrocks.tv/david-bowie-clipbizarre-et-beau-pour-lazarus/

Burrel, Romain. «Que vaut 'Lazarus', la pièce de David Bowie ?». Les Inrocks, 12 novembre 2016. Consulté le 15 juin 2017. <http://www.lesinrocks.com/2016/11/12/musique/que-vaut-lazaruspiece-de-david-bowie-11878951/>

Cachin, Olivier. « 'Blackstar' de David Bowie : l'album d'un génie torturé qui n'a plus rien à perdre ». L'Obs, 5 janvier 2016. Consulté le 15 juin 2017. <http://leplus.nouvelobs.com/ contribution/1466305-blackstar-de-david-bowie-l-album-d-un-genie-torture-qui-n-a-plus-rien-aperdre.html\#reaction>

Cassavetti, Hugo. « 'Blackstar', le clip étrange et perturbant de David Bowie ». Télérama, 20 novembre 2015. Consulté le 15 juin 2017. <http://www.telerama.fr/musique/blackstar-le-clipetrange-et-perturbant-de-david-bowie,134490.php>

Cassavetti, Hugo. « Blackstar, David Bowie ». Télérama, 4 janvier 2016. Consulté le 15 juin 2017. Consulté le 15 juin 2017. <http://www.telerama.fr/musiques/blackstar, 136357.php>

Clash. « Blackstar - David Bowie ». Rock Fever (blog), 11 janvier 2016. Consulté le 15 juin 2017. <http://clashdohertyrock.canalblog.com/archives/2016/01/11/33198929.html>

Cole, Jake. « David Bowie : Blackstar ». Spectrum Culture, January 11, 2016. Consulté le 15 juin 2017. <http://spectrumculture.com/2016/01/11/david-bowie-blackstar/>

Conte, Christophe. « On a écouté 'Blackstar' de David Bowie : un nouvel album dense et audacieux ». Les Inrocks, 14 décembre 2016. Consulté le 15 juin 2017. <http:// www.lesinrocks.com/2015/12/14/musique/notre-ecoute-en-avant-premiere-11793346/>

Cooper, Leonie. « How David Bowie told us he was dying in the 'Lazarus' video ». NME, January 11, 2016. Consulté le 15 juin 2017. <http://www.nme.com/blogs/nme-blogs/how-david-bowie-toldus-he-was-dying-in-the-lazarus-video-12143>

Coscarelli, Joe \& Michael Paulson. « David Bowie Allowed His Art to Deliver a Final Message ». The New York Times, Janaury 11, 2016.

Crockford, C.M. « David Bowie : Blackstar ». Punknews.org., January 12, 2016. Consulté le 15 juin 2017. <https://www.punknews.org/review/14116/david-bowie-blackstar>

Davet, Stéphane. « David Bowie sort de l'ombre avec 'Black Star', une chanson de dix minutes ». Le Monde, 20 novembre 2015. Consulté le 15 juin 2017. <http://www.lemonde.fr/musiques/ article/2015/11/20/david-bowie-fait-scintiller-black-star-une-chanson-de-10minutes_4814485_1654986.html>

Davet, Stéphane. « David Bowie, le Dorian Gray du rock ». L'Express, 22 sept. 2003. Consulté le 15 juin 2017. <https://www.lexpress.mu/article/david-bowie-le-dorian-gray-du-rock>

Dombal, Ryan. « David Bowie : Blackstar ». Pitchfork, 7 janvier 2016. Consulté le 15 juin 2017. <http://pitchfork.com/reviews/albums/21332-blackstar/>

Dooh. «Je tente David Bowie (Critique de Blackstar) ». Trop t'art (blog), 22 février 2016. Consulté le 15 juin 2017. <http://troptart.over-blog.com/2016/02/je-tente-david-bowie-critique-deblackstar.html> 
Duldig, Ingmar. « David Bowie : Blackstar ». AU., January 19, 2016. Consulté le 15 juin 2017. <http://music.theaureview.com/reviews/album-review-david-bowie-blackstar-2016-lp/> Embling. « David Bowie : Blackstar ». Tiny Mix Tapes. Consulté le 15 juin 2017. <https:// www.tinymixtapes.com/music-review/david-bowie-blackstar>

Fricke, David. « David Bowie : Blackstar ». Rolling Stone, December 23, 2015. Consulté le 15 juin 2017. <http://www.rollingstone.com/music/albumreviews/david-bowie-blackstar-20151223> Gerard, Chris. « David Bowie : Blackstar ». PopMatters, 8 January 2016. Consulté le 15 juin 2017. <http://www.popmatters.com/review/david-bowie-blackstar/>

Gerhart, Thompson D. « David Bowie : Blackstar ». Sputnik Music, January 17, 2016. Consulté le 15 juin 2017. <http://www.sputnikmusic.com/review/69534/David-Bowie-Blackstar/>

Gill, Andy. «David Bowie new album Blackstar, review : A Bowie desperate to break with the past ». The Independent, January 8, 2016. Consulté le 15 juin 2017. <http://

www.independent.co.uk/arts-entertainment/music/reviews/david-bowies-blackstar-exclusivefirst-review-a-bowie-desperate-to-break-with-the-past-a6783456.html>

Gill, Andy. « David Bowie's Blackstar - exclusive first review : A Bowie desperate to break with the past. » The Independent, 28 December 2015. Consulté le 15 juin 2017. <http:// www.independent.co.uk/arts-entertainment/music/reviews/david-bowies-blackstar-exclusivefirst-review-a-bowie-desperate-to-break-with-the-past-a6783456.html>

Greene, Andy. The Inside Story of David Bowie's Stunning New Album, 'Blackstar'. Rolling Stone, November 23, 2015. Consulté le 15 juin 2017. <http://www.rollingstone.com/music/features/theinside-story-of-david-bowies-stunning-new-album-blackstar-20151123>

Griggs, Brandon. « 'Blackstar' : Haunting final album hints at David Bowie's death ». CNN, January 13, 2016. Consulté le 15 juin 2017. <http://edition.cnn.com/2016/01/11/entertainment/davidbowie-blackstar-lazarus-death-meaning-feat/index.html>

Guillot, Antoine. « Les mystères de l'étoile noire ». France Culture, 26 novembre 2015. Consulté le 15 juin 2017. https://www.franceculture.fr/emissions/revue-de-presse-culturelle-dantoineguillot/les-mysteres-de-letoile-noire

Harman, Duncan. « David Bowie : Blackstar ». The Skinny, January 12, 2016. Consulté le 15 juin 2017. http://www.theskinny.co.uk/music/reviews/albums/david-bowie-blackstar

Holland, Eric. « Producer Tony Visconti Talks David Bowie and Blackstar ». Hollandude, January 6, 2016. Consulté le 15 juin 2017. <http://hollandude.com/producer-tony-visconti-talks-davidbowie-and-blackstar/>

Jonze, Tim. « Was David Bowie saying goodbye on Blackstar ? ». The Guardian, January 11, 2016. Consulté le 15 juin 2017. <https://www.theguardian.com/music/musicblog/2016/jan/11/wasdavid-bowie-saying-goodbye-on-blackstar>

Koechlin, Stéphane. « David Bowie, mort du Dorian Gray de la musique pop ». Valeurs Actuelles, 14 janvier 2016. Consulté le 15 juin 2017. <http://www.valeursactuelles.com/david-bowie-mort-dudorian-gray-de-la-musique-pop-58479>

Langs, Bester. « La vie après Bowie ». Gonzaï, 13 janvier 2016. Consulté le 15 juin 2017. <http:// gonzai.com/la-vie-apres-bowie/>

Lazimi, Charlotte. « Blackstar : la dernière Étoile noire de David Bowie ». L'Express, 18 décembre 2015. Consulté le 15 juin 2017. <http://www.lexpress.fr/culture/musique/blackstar-la-nouvelleetoile-noire-de-david-bowie_1747246.html> 
Leorne, Ana. « Dear Dr. Freud-David Bowie Hits the Couch : A psychoanalytical approach to some of his personae », in David Bowie : Critical Perspectives. Ed. Eoin Devereux, et al. New York : Routledge, 2015.

Mathers, Ian. « David Bowie : Blackstar ». Dusted, January 12, 2016. <http:// dustedmagazine.tumblr.com/post/137159122229/david-bowie-isorcacolumbia>

McCormick, Neil. « David Bowie, Blackstar, review : 'extraordinary'. » The Daily Telegraph, 8 January 2016. Consulté le 15 juin 2017. <http://www.telegraph.co.uk/music/what-to-listen-to/ david-bowie-blackstar-first-listen-extraordinary/>

McCormick, Neil. « David Bowie's new song, Blackstar, review : 'Major Tom is dead. Bowie lives' . The Telegraph, November 20, 2015. <http://www.telegraph.co.uk/music/what-to-listen-to/davidbowies-new-song-blackstar-review-major-tom-is-dead-bowie-l/>

Nadeau, Louis. « 'Blackstar', les éclats noirs de David Bowie ». La Croix, 8 janvier 2016. Consulté le 15 juin 2017. <http://www.la-croix.com/Culture/Musique/Blackstar-les-eclats-noirs-de-DavidBowie-2016-01-08-1401542>

Nuc, Olivier. « Blackstar de David Bowie, entre new wave et jazz expérimental ». Le Figaro, 18 décembre 2015, Consulté le 15 juin 2017. <http://www.lefigaro.fr/musique/ 2015/12/17/03006-20151217ARTFIG00275-nous-avons-ecoute-blackstar-le-nouvel-album-dedavid-bowie.php>

Pareles, Jon. « Review : ‘Blackstar,' David Bowie's Emotive and Cryptic New Album ». The New York Times, January 6, 2016. Consulté le 15 juin 2017. <https://www.nytimes.com/2016/01/07/arts/ music/review-blackstar-david-bowies-emotive-and-cryptic-new-album.html ?mcubz=2>

Payne, Chris. « David Bowie's Final Album ‘Blackstar' \& 'Lazarus' Video Were Goodbye Notes ». Billboard, January 11, 2016. Consulté le 15 juin 2017. <http://www.billboard.com/articles/ columns/rock/6836563/david-bowie-blackstar-goodbye-note-death-lazarus-2016>

Péron, Didier et Julien Gester. « La dernière mort de David Bowie ». Libération, 11 janvier 2016. Consulté le 15 juin 2017. <http://next.liberation.fr/culture-next/2016/01/11/la-derniere-mortde-david-bowie_1425797>

Petridis, Alexis. « David Bowie : Blackstar review - a spellbinding break with his past ». The Guardian, Janaury 7, 2016. Consulté le 15 juin 2017. <https://www.theguardian.com/music/2016/ jan/07/david-bowie-blackstar-review-a-spellbinding-break-with-his-past>

Pliskin, Fabrice. « Blackstar : la pop gargantuesque de Bowie. » L'Obs, 8 janvier 2016. Consulté le 15 juin 2017. <http://tempsreel.nouvelobs.com/culture/20160105.OBS2239/blackstar-la-popgargantuesque-de-bowie.html>

Pottier, Jean-Marie. « Notre deuil de Bowie ne fait pas de nous d'incurables nostalgiques ». Slate, 18 janvier 2016. Consulté le 15 juin 2017. <http://www.slate.fr/story/112767/deuil-bowienostalgie>

Rancic, Michael. « David Bowie - Blackstar. » Exclaim !, 7 January 2016. Consulté le 15 juin 2017. <http://exclaim.ca/music/article/david_bowie-_blackstar>

Rayner, Ben. « David Bowie's Blackstar a nearly perfect goodbye : review ». Toronto Star, January 8, 2016. Consulté le 15 juin 2017. <https://www.thestar.com/entertainment/music/2016/01/08/ david-bowie-aims-high-with-blackstar-review.html>

Richard, Philippe. « Blackstar, le dernier album de David Bowie, morceau par morceau ». OuestFrance, 8 janvier 2016. Consulté le 15 juin 2017. <http://www.ouest-france.fr/culture/musiques/ bowie-blackstar-morceau-par-morceau-3958759> 
Richards, Sam. « David Bowie - Blackstar review ». NME. Consulté le 15 juin 2017. <http:// www.nme.com/reviews/album/reviews-david-bowie-16363>

Rodgers, Jude. «The final mysteries of David Bowie's Blackstar - Elvis, Crowley and 'the villa of Ormen ». The Guardian, January 21, 2016. Consulté le 15 juin 2017. <https:// www.theguardian.com/music/2016/jan/21/final-mysteries-david-bowie-blackstar-elvis-crowleyvilla-of-ormen>

Schiffer, Daniel Salvatore. « David Bowie, de Ziggy Stardust à Blackstar : la mort d'un dandy stellaire », Médiapart, 12 janvier 2016. Consulté le 15 juin 2017. <https://blogs.mediapart.fr/ daniel-salvatore-schiffer/blog/120116/david-bowie-de-ziggy-stardust-blackstar-la-mort-dundandy-stellaire>

Sébastien. «Blackstar - David Bowie - Critique ». Les Critiques Musicales - blog, 14 avril 2016. Consulté le 15 juin 2017. <https://critiquesmusicalesblog.wordpress.com/2016/04/14/blackstardavid-bowie/>

Siclier, Sylvain. « 'Blackstar', le dernier album de David Bowie ». Le Monde, 8 janvier 2016. Consulté le 15 juin 2017. <http://www.lemonde.fr/musiques/article/2016/01/08/david-bowie-al-age-des-collages_4843662_1654986.html>

Soto, Alfred. « Review : David Bowie Remains the Original Starman on 'Blackstar' « . Spin, January 6, 2016. Consulté le 15 juin 2017. <http://www.spin.com/2016/01/review-david-bowie-blackstar/ $>$

Staff. « David Bowie finally tops US Billboard charts with Blackstar ». The Guardian, January 18, 2016. Consulté le 15 juin 2017. <<https://www.theguardian.com/music/2016/jan/18/davidbowie-finally-tops-us-billboard-charts-with-blackstar>

Staff. « The A.V. Club's 20 Best Albums of 2016 ». The A.V. Club, December 12, 2016. Consulté le 15 juin 2017.<http://www.avclub.com/article/v-clubs-20-best-albums-2016-246644>

Tabakis, Peter. « David Bowie's Beautiful Goodbye ». Pretty Much Amazing, January 12, 2016. Consulté le 15 juin 2017. <https://prettymuchamazing.com/features/david-bowies-beautifulgoodbye>

Thompson, Erik. « David Bowie bids Earth farewell with a wonderfull album ». The Line of Best Fit, January 11, 2016. Consulté le 15 juin 2017. <https://www.thelineofbestfit.com/reviews/albums/ david-bowie>

Tonnet, Auréliano. « Bowie, de vive voix ». Le Monde, 12 janvier 2016. Consulté le 15 juin 2017file ://localhost/. <http/ : :www.lemonde.fr :culture :article: 2016: 01: 12 :bowie-de-vivevoix_4845525_3246.html>

Vincent, Alice. « David Bowie dies from cancer aged 69 : tributes, memories and pictures ». The Telegraph, January 12, 2016. Consulté le 15 juin 2017. <http://www.telegraph.co.uk/music/news/ david-bowie-dies-from-cancer-aged-69-tributes-memories-and-pictu/>

Vincent, Alice. « Was David Bowie's Blackstar named after a cancer lesion? » The Telegraph, January 12, 2016. Consulté le 15 juin 2017. <http://www.telegraph.co.uk/music/news/was-davidbowies-blackstar-named-after-a-cancer-lesion/>

Vishnevetsky, Ignatiy. " David Bowie goes noir with his intoxicating Blackstar ». A.V. Club, January 8, 2016. Consulté le 15 juin 2017. <http://www.avclub.com/review/david-bowie-goesnoir-intoxicating-blackstar-230272?permalink=true> 
Viviant, Arnaud. « Mort De David Bowie : Blackstar, ‘Un Album Testament'« . LCI, 11 janvier 2016. Consulté le 15 juin 2017. <http://www.lci.fr/france/mort-de-david-bowie-blackstar-un-albumtestament-1243831.html>

Watson, Tom. « David Bowie : Blackstar ». Crack Magazine. Consulté le 15 juin 2017. <http:// crackmagazine.net/article/music/david-bowie-blackstar/>

Wroble, Jonathan. « David Bowie : Blackstar ». Slant, January 11, 2016. Consulté le 15 juin 2017. <http://www.slantmagazine.com/music/review/david-bowie-blackstar>

\section{Sources secondaires}

Barros, Paula. «'Piety to a dead man' : les limites de la consolation dans la pratique épistolaire de Sir Kenelm Digby (Angleterre, 1633) ». Exercices de rhétorique [En ligne], 9 | 2017, mis en ligne le 21 juin 2017, consulté le 21 juin 2017. <http://rhetorique.revues.org/543>

Bossuet, Jacques-Bénigne. Cuvres, édition de l'abbé B. Vélat et Yvonne Champaillé. Paris : Gallimard, Bibliothèque de la Pléiade, 1961.

Brosig, B. « The Dorian Gray Syndrome : psychodynamic need for hair growth restorers and other fountains of youth ». Paper presented at the Continuous Medical Education Board of the Landesärztekammer Hessen, Clinical Pharmacology Section, on 29. 4. in Bad Nauheim, FRG, 2000.

Cicéron, « De L'orateur », Livre Deux, LXXXV. Cuvres Complètes. Ed. J.V. Leclercq. Paris : Lefèvre, 1821.

Foucault, Michel. Les Hétérotopies, le corps utopique. Fécamp : Lignes, 2009.

Hache, Sophie. «L'amplification dans les oraisons funèbres de Fléchier : l'éclat de l'épithète ", Exercices de rhétorique [En ligne], 4 | 2014, mis en ligne le 28 septembre 2015, consulté le 7 juin 2017. URL : <http://rhetorique.revues.org/347>

Hache, Sophie. «Le style de l'histoire dans l'« Oraison funèbre de Condé » de Bossuet », Dixseptième siècle, 2009/4 (n²45), p. 703-716. DOI : 10.3917/dss.094.0703. Consulté le 7 juin 2017. <http://www.cairn.info/revue-dix-septieme-siecle-2009-4-page-703.htm>

Jodra, Serge. Imago Mundi. Consulté le 15 juin 2017. <http://www.cosmovisions.com/ textOraison.htm>

Leorne, Ana. « Dear Dr. Freud-David Bowie Hits the Couch : A psychoanalytical approach to some of his personae », in David Bowie : Critical Perspectives. Ed. Eoin Devereux, et al. New York : Routledge, 2015

Martin-Ulrich, Claudie. « Présentation : consolation et rhétorique ». Exercices de rhétorique [En ligne], 9 | 2017, mis en ligne le 21 juin 2017, consulté le 21 juin 2017. <http:// rhetorique.revues.org/543>

Molinié, George et Jean Mazaleyrat, Vocabulaire de la stylistique. Paris : PUF, 1989.

Noille, Christine. «La rhétorique de l'oraison funèbre : présentation, traduction, exemples ", PFSCL (Papers on French Seventeenth Century Literature), vol. XLII, no. 82, 2015, 41-67.

Pellisson-Fontanier, Paul. « Projet de l'histoire de Louis XIV », CEuvres diverses. Genève : Slatkine Reprints, 1735.

Powers, Ann. « Mourning Stars Through Streams, Screens And Communities. On Music And Collective Grief In $2016 »$. NPR, December 29, 2016. Consulté le 15 juin 2017. <http:// www.npr.org/sections/therecord/2016/12/29/507146995/when-departed-stars-become-digitalsaints-on-music-and-collective-grief-in-2016> 
Schiffer, Daniel Salvatore. Petit éloge de David Bowie : le dandy absolu. Paris : Les Nouvelles Éditions François Bourrin, 2016.

Shishigami. Consulté le 15 juin 2017. <http://www.shishigami.com/srfa/saintbowie.html>

\section{NOTES}

1. Pour rappel, dans The Picture of Dorian Gray qu'Oscar Wilde publie à Londres en 1890 dans Lippincott's Monthly Magazine, un jeune homme narcissique de grande beauté éprouve une forme de jalousie à l'égard de son propre portrait, au point qu'il exprime le souhait que son portrait vieillisse à sa place, afin de préserver sa jeunesse et sa beauté. Le dénouement a lieu une vingtaine d'années plus tard, lorsque Dorian Gray transperce d'un coup de poignard le tableau qui retrouve aussitôt son état initial tandis que le protagoniste meurt et que son visage se transforme en celui d'un vieillard monstrueux.

2. L'expression "syndrome de Dorian Gray» a été suggérée par Brosig pour décrire un phénomène socio-culturel et un état psychiatrique qui, se développent le plus souvent chez les hommes, et se manifestent par un souci extrême de l'apparence et des capacités physiques ainsi que par une inquiétude liée au vieillissement (Brosig 2000).

3. Freddy Mercury avait cherché à cacher son épuisement et les effets les plus visibles de la maladie à grand renfort de maquillage.

4. Chez la plupart des critiques de langue française, le terme "pop» désigne, plus qu'un style proprement dit ou une période, des visées moins ambitieuses et plus commerciales, des formes plus accessibles au grand public. Les connotations du terme peuvent être différentes aux ÉtatsUnis et en Grande-Bretagne.

5. Voir Jacques-Bénigne Bossuet. CEuvres, édition de l'abbé B. Vélat et Yvonne Champaillé. Paris : Gallimard, Bibliothèque de la Pléiade, 1961.

6. Le vocabulaire utilisé par les fans sur les réseaux sociaux est bien sûr éloigné de celui des journalistes professionnels, mais la teneur des propos est comparable.

7. Je remercie Claudie Martin-Ulrich pour son aide documentaire sur l'oraison funèbre et la consolation.

\section{RÉSUMÉS}

Cet article s'intéresse aux critiques françaises, britanniques et américaines de l'ultime album de David Bowie, Blackstar. Élogieuses pour la plupart, elles envisagent l'album comme une mise en son et en image par l'artiste de son propre décès. Je m'interroge sur le ton adopté par la presse française et la presse anglophone. Comment rendent-elles compte de l'organisation esthétique de sa mort conçue par David Bowie ? Quels commentaires livrent-elles sur une œuvre qui parle des derniers instants et de l'après par le biais d'un rock avant-gardiste ? Y voient-elles l'expression d'une mort moderne, une mort rock ? Ou leurs emprunts aux genres de l'oraison funèbre et de la consolation révèlent-ils d'autres possibilités ?

This article focuses on the French, British, and American reviews of David Bowie's ultimate album, Blackstar. Almost unanimously positive, they consider the album as the staging by the 
artist of his own demise. I analyze the tone adopted by the French and English speaking press. What account do they provide of Bowie's unprecedented aesthetic organization of his death? How do they comment on an artwork that deals with life's final moments and what comes next by means of avant-garde rock? Do they consider it as an expression of modern death? Or do their borrowings from such genres as the funeral oration or the consolation point to other directions?

\section{INDEX}

Mots-clés : David Bowie, Blackstar, mort, oraison funèbre, consolation, critiques

Keywords : David Bowie, Blackstar, death, funeral oration, consolation, reviews

\section{AUTEURS}

\section{CLAUDE CHASTAGNER}

Professeur

Université Paul Valéry-Montpellier

claude.chastagner@univ-montp3.fr 